\title{
Drug information update: paliperidone palmitate for schizophrenia
}

\author{
Abraham M. Nussbaum, ${ }^{1,2}$ T. Scott Stroup ${ }^{3}$
}

The Psychiatrist (2013), 37, 164-166, doi: 10.1192/pb.bp.113.042739

${ }^{1}$ Behavioral Health Service, Denver Health, Denver, Colorado, USA; ${ }^{2}$ University of Colorado, Aurora, USA ${ }^{3}$ Columbia University, New York, USA Correspondence to Abraham M. Nussbaum (abraham.nussbaum@dhha.org) First received 11 Jan 2013, accepted 28 Jan 2013

\begin{abstract}
Aims and method To review the evidence for the use of paliperidone palmitate for people with schizophrenia and schizophrenia-like illnesses. We searched the Cochrane Schizophrenia Group Specialised Register and contacted the manufacturer of paliperidone palmitate, the US Food and Drug Administration, and the authors of papers that reported study results.
\end{abstract}

Results Based on the evidence from five short-term, placebo-controlled studies, paliperidone palmitate is efficacious as an antipsychotic. Its adverse effects are similar to those of the closely related compounds paliperidone and risperidone.

Extrapyramidal side-effects, weight gain and tachycardia are more common with paliperidone palmitate than placebo. Paliperidone palmitate was associated with substantial increases in serum prolactin but not with increased sexual side-effects in these studies. In two studies paliperidone palmitate was similar to depot risperidone.

Clinical implications Paliperidone palmitate is an effective antipsychotic whose optimal dose appears to be between 39 and $234 \mathrm{mg}$ every 4 weeks. We have no data assessing its long-term effectiveness or comparing it with any long-acting injected antipsychotic other than depot risperidone.

Declaration of interest A.M.N. receives funding from the University of Chicago Program on Medicine and Religion for unrelated projects. T.S.S. receives funding from Agency for Healthcare Research and Quality and the National Institute of Mental Health for unrelated projects.
Antipsychotic treatment of schizophrenia and schizophrenialike illnesses is associated with reduced psychotic symptoms, an increased time to recurrence of psychotic symptoms, and reduced rates of hospital admissions. ${ }^{1}$ However, people diagnosed with schizophrenia often do not fully adhere to oral medication regimens. ${ }^{2}$ In an effort to improve adherence, physicians often recommend long-acting intramuscular antipsychotic injections. ${ }^{3}$

Pharmaceutical manufacturers typically prepare longacting intramuscular formulations of antipsychotics as esters, suspended in oil. These formulations are absorbed and eliminated by the body at a slower rate than oral preparations. When it produced the long-acting formulation of risperidone, the manufacturer did not esterify the drug. They instead produced an aqueous suspension of risperidone microspheres. As a result, long-acting injections of risperidone must be refrigerated, reconstituted with a diluent supplied by the manufacturer, and administered within 6 hours of reconstitution. In addition, clinicians need to administer the risperidone long-acting formulation bi-weekly to maintain a therapeutic concentration. ${ }^{4}$

\section{Paliperidone}

Paliperidone is the active metabolite of risperidone. Cytochrome P450 enzymes 2D6 and 3A4 metabolise risperidone to 9 -hydroxyrisperidone, ${ }^{5}$ marketed orally as paliperidone. We previously reviewed its efficacy and safety for the Cochrane Collaboration. ${ }^{6}$ To make a long-acting injectable formulation of paliperidone, its hydroxyl group is esterified. Paliperidone palmitate is an aqueous suspension with low water solubility that is absorbed and eliminated slowly. It is supplied in pre-filled syringes of 39, 78, 117, 156 or $234 \mathrm{mg}$. Clinicians reconstitute it with vigorous shaking before administration. Paliperidone palmitate does not require refrigeration and can be administered monthly. After intramuscular administration, the drug slowly dissolves. After hydrolysis to paliperidone, it becomes available in the systemic circulation. The drug is measured in plasma as early as the first day of administration and lasts as long as 126 days, reaching maximum plasma concentration at 13 days. ${ }^{7}$ We have previously reviewed its efficacy and safety for the Cochrane Collaboration. ${ }^{8}$

\section{Method}

\section{Search methods}

We searched the Cochrane Schizophrenia Group Specialised Register for randomised controlled trials (RCTs) comparing paliperidone palmitate with any other treatment for schizophrenia. We inspected references of identified studies 
for further trials and contacted the manufacturers of paliperidone palmitate, the US Food and Drug Administration and authors of relevant reports for additional material. We used RevMan version 5.1 software on Windows to conduct the analyses. All relevant RCTs were included.

\section{Data collection and analysis}

Data were analysed on an intention-to-treat basis. When appropriate, we calculated risk ratios (RR) and 95\% confidence intervals with the number needed to benefit/ harm statistic $(\mathrm{NNB} / \mathrm{NNH})$. We calculated weighted mean differences (WMD) for continuous data.

\section{Results}

We included five studies ${ }^{9-13}$ with 2215 participants that compared paliperidone palmitate with placebo. Fewer people randomised to paliperidone palmitate left studies early $(n=2183,5$ RCTs: $\mathrm{RR}=0.76,95 \%$ CI $0.70-0.84$; NNB 9, 95\% CI 7-14). People receiving any dose of paliperidone palmitate were less likely to show no improvement in global state $(n=1696,4$ RCTs: $\mathrm{RR}=0.79,95 \%$ CI $0.74-0.85$; NNB 7, 95\% CI 5-9). In a single trial designed to study recurrence of psychosis, people randomised to paliperidone palmitate were less likely to experience a recurrence than those randomised to placebo $(n=312: \mathrm{RR}=0.28,95 \%$ CI $0.17-0.48$; NNB 5, 95\% CI 4-6). People who received paliperidone palmitate were also less likely to experience a recurrence of psychotic symptoms in studies that recorded recurrence as an adverse event $(n=1837,4$ RCTs: $\mathrm{RR}=0.55$, 95\% CI $0.44-$ 0.68; NNB 10, 95\% CI 8-14). Paliperidone palmitate was associated with less use of anxiolytics $(n=2170,5$ RCTs: $\mathrm{RR}=0.89,95 \% \mathrm{CI} 0.83-0.96$; NNB 16, 95\% CI 11-44) and with fewer reports of agitation or aggression $(n=2180,5$ RCTs: $\mathrm{RR}=0.65$, 95\% CI 0.46-0.91; NNB 39, 95\% CI 25150). Both men and women who received paliperidone palmitate experienced significant elevation in serum prolactin $(\mathrm{ng} / \mathrm{mL})$, but the data were too heterogeneous for us to analyse. We found no evidence of sexual dysfunction associated with paliperidone palmitate in these short-term trials, but people receiving paliperidone palmitate gained more weight than those who received placebo ( $n=2052,5$ RCTs: WMD 1.34, 95\% CI 0.97-1.70).

\section{Paliperidone palmitate $\boldsymbol{v}$. depot risperidone}

Two additional studies ${ }^{14,15}$ compared paliperidone palmitate with depot risperidone $(n=1969)$. In these flexibly dosed trials, the mean doses of paliperidone palmitate were $73.3 \mathrm{mg}$ and $104.6 \mathrm{mg}$ every 4 weeks compared with depot risperidone at mean doses of $35.3 \mathrm{mg}$ and $31.7 \mathrm{mg}$ every 2 weeks. There was no difference for leaving these studies early for any reason between paliperidone palmitate and depot risperidone $(n=1969,2$ RCTs: $\mathrm{RR}=1.12$, 95\% CI 1.00-1.25). Those receiving paliperidone palmitate were no more likely to have a recurrence of psychotic symptoms ( $n=1961,2$ RCTs: $\mathrm{RR}=1.23,95 \%$ CI $0.98-1.53)$. There were a total of six deaths in these two trials, with five in the paliperidone palmitate groups. Although this difference was not significant $(n=1967,2$ RCTs: $R R=3.62,95 \%$ CI
0.60-21.89), the small number of these events and wide confidence interval makes it unclear whether this finding is meaningful. Patients randomised to paliperidone palmitate were significantly less likely to use anticholinergic medications $(n=1587,2$ RCTs: $\mathrm{RR}=0.67,95 \%$ CI $0.55-0.82$; NNB 13, 95\% CI 10-24).

We found no data regarding service use, quality of life, patient satisfaction or cost. Similarly, we found no randomised studies that compared paliperidone palmitate with any other treatments for schizophrenia or schizophrenia-like illnesses.

\section{Discussion}

In short-term studies, paliperidone palmitate is an efficacious antipsychotic drug. Its adverse effects are similar to oral paliperidone, oral risperidone and depot risperidone. Extrapyramidal side-effects, weight gain and tachycardia are more common with paliperidone palmitate than placebo. Paliperidone palmitate is associated with substantial increases in serum prolactin. Used at mean doses of approximately $70-110 \mathrm{mg}$ every 4 weeks, it appears comparable in efficacy and tolerability with depot risperidone used at mean doses of approximately $35 \mathrm{mg}$ every 2 weeks. ${ }^{8,16}$ People who received paliperidone palmitate were significantly less likely to receive anticholinergic medications. Paliperidone palmitate has two practical advantages over depot risperidone in that it does not need to be refrigerated and can be administered monthly rather than bi-weekly, but we found no clear benefit of paliperidone palmitate over depot risperidone.

This study confirms our suspicion that paliperidone palmitate will affect people with schizophrenia similarly to depot risperidone. For individual clinicians to select paliperidone palmitate over other long-acting injectable antipsychotics on the basis of appropriate evidence, researchers will need to conduct longer-term pragmatic studies that more closely resemble the lived experience of patients with schizophrenia. Appropriate comparators include fluphenazine decanoate, haloperidol decanoate, depot olanzapine and depot risperidone. Clinicians would also benefit from studies that assess the efficacy, adverse effects and safety of long-term use of paliperidone palmitate, including behaviour, mortality, satisfaction with treatment and cost-effectiveness in comparison with oral antipsychotics.

\section{Acknowledgements}

We thank our respective departments and the Cochrane Schizophrenia Group for their support. See also Nussbaum \& Stroup. ${ }^{8}$

\section{About the authors}

Abraham M. Nussbaum, Director, Adult Inpatient Psychiatry, Behavioral Health Service, Denver Health, Denver, and University of Colorado, USA. T. Scott Stroup, Director, Program for Intervention Effectiveness Research, New York State Psychiatric Institute, and Columbia University, New York, USA. 


\section{References}

1 Freedman R. Schizophrenia. N Engl J Med 2003; 349: 1738-49.

2 Lieberman JA, Stroup TS, McEvoy JP, Swartz MS, Rosenheck RA, Perkins DO, et al. Effectiveness of antipsychotic drugs in patients with chronic schizophrenia. N Engl J Med 2005; 353: 1209-23.

3 McEvoy JP. Risks versus benefits of different types of long-acting injectable antipsychotics. J Clin Psych 2006; 67: 15-8.

4 Janssen Pharmaceutica Products LP. Risperdal Consta (Risperidone) LongActing Injection Prescribing Information. Janssen Pharmaceutica Products LP, 2003.

5 Fang J, Bourin M, Baker GB. Metabolism of risperidone to 9-hydroxyrisperidone by human cytochromes P450 2D6 and 3A4. Naunyn Schmiedebergs Arch Pharmacol 1999; 359: 147-51.

6 Nussbaum AM, Stroup TS. Oral paliperidone for schizophrenia. Cochrane Database Syst Rev 2009; CD006369.

7 Janssen. Invega Sustenna (Paliperidone Palmitate) Extended-Release Injectable Suspension Prescribing Information. Orth-McNeil-Janssen Pharmaceuticals, 2009.

8 Nussbaum AM, Stroup TS. Paliperidone palmitate for schizophrenia. Cochrane Database Syst Rev 2012; CD008296.

9 Gopal S, Hough DW, Xu H, Lull JM, Gassmann-Mayer C, Remmerie BM et al. Efficacy and safety of paliperidone palmitate in adult patients with acutely symptomatic schizophrenia: a randomized, double-blind, placebo-controlled, dose-response study. Int Clin Psychopharmacol 2010; 25: 247-56.
10 Hough D, Gopal S, Vijapurkar U, Lim P, Morozova M, Eerdekens M. Paliperidone palmitate maintenance treatment in delaying the time-torelapse in patients with schizophrenia: a randomized, double-blind, placebo-controlled study. Schizophr Res 2010; 116: 107-17.

11 Kramer M, Litman R, Hough D, Lane R, Lim P, Liu Y, et al. Paliperidone palmitate, a potential long-acting treatment for patients with schizophrenia. Results of a randomized, double-blind, placebocontrolled efficacy and safety study. Int J Neuropsychopharmacol 2009; 13: $635-47$

12 Nasrallah HA, Gopal S, Gassmann-Mayer C, Quiroz JA, Lim P, Eerdekens $M$, et al. A controlled, evidence-based trial of paliperidone palmitate, a long-acting injectable antipsychotic, in schizophrenia. Neuropsychopharmacol 2010; 10: 2072-82.

13 Pandina GJ, Lindenmayer JP, Lull J, Lim P, Gopal S, Herben V, et al. A randomized, placebo-controlled study to assess the efficacy and safety of 3 doses of paliperidone palmitate in adults with acutely exacerbated schizophrenia. J Clin Psychopharmacol 2010; 30: 235-44.

14 Fleischhacker WW, Gopal S, Lane R, Gassmann-Mayer C, Lim P, Hough D, et al. A randomized trial of paliperidone palmitate and risperidone longacting injectable in schizophrenia. Int J Neuropsychopharmacol 2011; Jul 22: 1-12. (Epub ahead of print.)

15 Pandina G, Lane R, Gopal S, Gassmann-Mayer C, Hough D, Remmerie B et al. A double-blind study of paliperidone palmitate and risperidone long-acting injectable in adults with schizophrenia. Prog Neuropsychopharmacol Biol Psychiatry 2011; 35: 218-26.

16 Nussbaum AM, Stroup TS. Paliperidone palmitate for schizophrenia Schiz Bull 2012; 38: 1124-7. 